\title{
SURFACE PARAMETERS ESTIMATION USING RADARSAT-2 POLARIMETRIC DATA OVER WHEAT COVERED AREA
}

\author{
Quan Chen ${ }^{(1)}$,Zhen Li ${ }^{(1)}$, Aimin Cai ${ }^{(2)(3)}$, Bangsen Tian ${ }^{(1)(2)}$ \\ (1) Center for Earth Observation and Digital Earth, Chinese Academy of Sciences \\ (2) Graduate University of Chinese Academy of Sciences \\ (3) State Key Laboratory of Remote Sensing Science, Jointly Sponsored by the Institute of Remote \\ Sensing Applications of Chinese Academy of Sciences and Beijing Normal University \\ Beijing, 100101, China, Email: qchen@ceode.ac.cn
}

\section{INTRODUCTION}

In the field of agriculture, robust harvests and crop yields are challenged by the dynamic nature of soil and crop conditions that fluctuate throughout the growing season. Satellite SAR imagery is an efficient method for mapping crop and underground soil characteristics over large spatial areas and tracking temporal changes in soil and crop conditions. SAR sensors such as RADARSAT-1 and ERS can only provide single radar image, ENVISAT/ASAR can provide two polarizations images at three different acquirements, but only $\mathrm{HH} / \mathrm{VV}$ is used effectively due to low signal noise ratio of cross-polarization ( $\mathrm{HV}$ or $\mathrm{VH}$ ) of ASAR. Consequently, more than one acquisition date is usually required to estimate meaningful crop and soil information using these conventional sensor data. RADARSAT-2's powerful new features in terms of polarization, spatial resolution, look direction and orbit control, which can benefit the agricultural sector. In the quad-polarized mode of RADARSAT-2, some important and meaningful polarimetric parameters can be obtained, in addition to four different polarization channels. More valuable crop and soil information can be extracted from one RADARSAT-2 image, thus eliminating the need for data acquisition over the course of several dates.

\section{EXPERIMENT AND DATA}

In order to develop methods for monitoring soil and crop state using C-band polarimetric data of Radarsat-2, two images both at quad-polarized mode are acquired at 26th April and 26th May, 2009, respectively, corresponding to the Booting Stage and Milking Stage of wheat phenology. The images covered Dingxing county, which is the main agricultural county in the middle part of Hebei plain. 


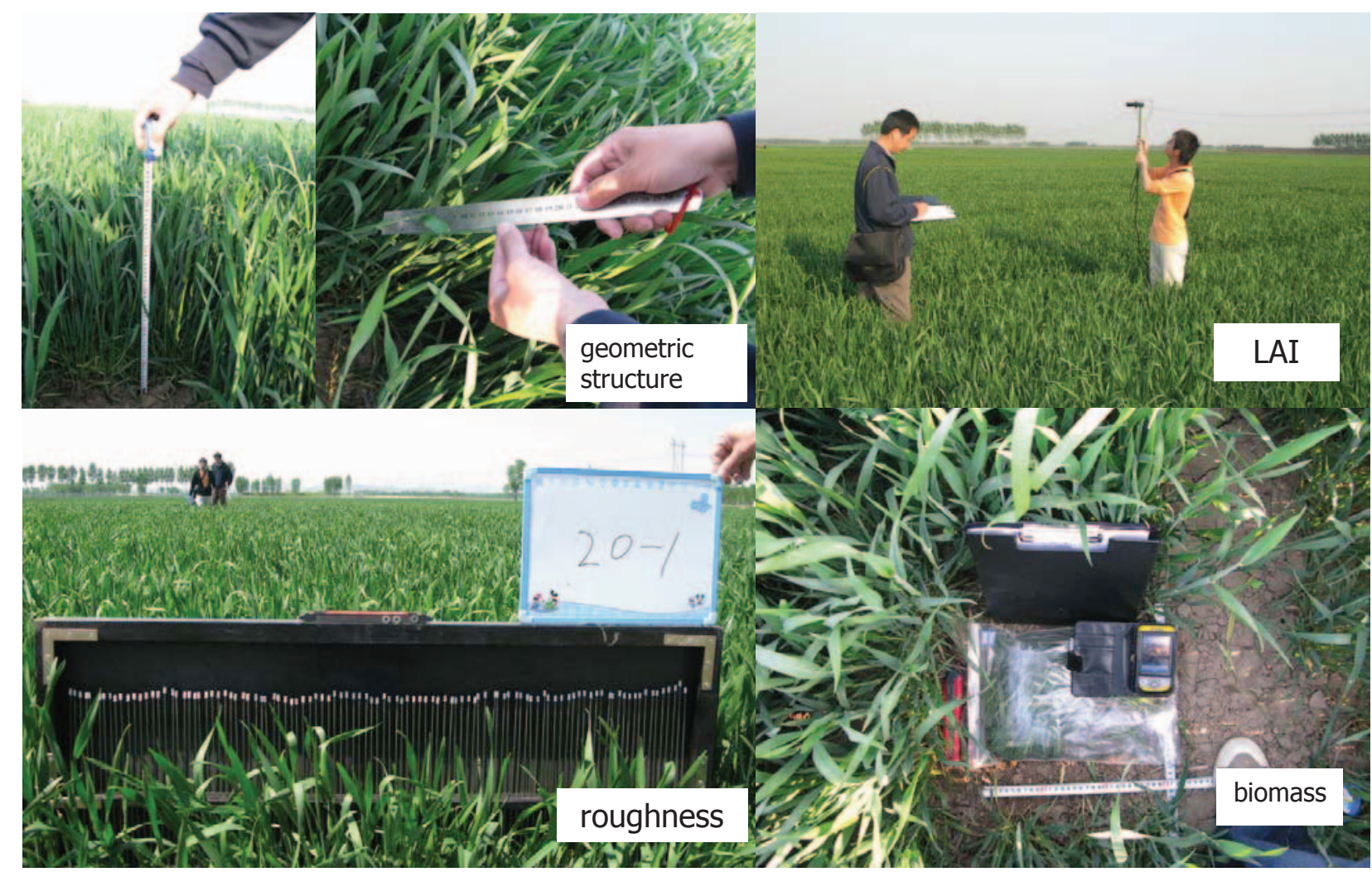

Fig.1 The pictures of field work

In the mean time of satellite SAR data acquired, ground experiments and in-situ samples were employed over wheat covered area at total 50 selected sampling points, many soil and wheat parameters are sampled, including soil moisture, surface roughness, wheat height, LAI, leave biomass, stem biomass, plant density, leave geometric structure, et al.. The pictures of field work are shown in Fig.1. For analyzing wheat characteristics dynamic throughout its whole life circle, samplings are also executed in its all important twelve phonological periods in four selected points around Gucheng agricultural meteorological station, which is a national experimental center located in our study area. The twelve phenological periods are Three-leaf Stage, Tillering Stage, Stop-Anagen Stage, Turning Green Stage, Double Ridge Stage, Jointing Stage, Booting Stage, Heading Stage, Flowering Stage, Milking Stage, Dough Stage and Mature Stage, range from Oct..2008 to Aug. 2009. In the western one of the four points, another wheat field is conversed as Non-Irrigation Field over its growth circle, which means only nature rainfall available for this field, for comparing with the others to analysis how drought effects on wheat's biomass and final product (The products of these five fields are also collected). The comparison of biomass through wheat life circle of these five fields are shown in Fig.2. 


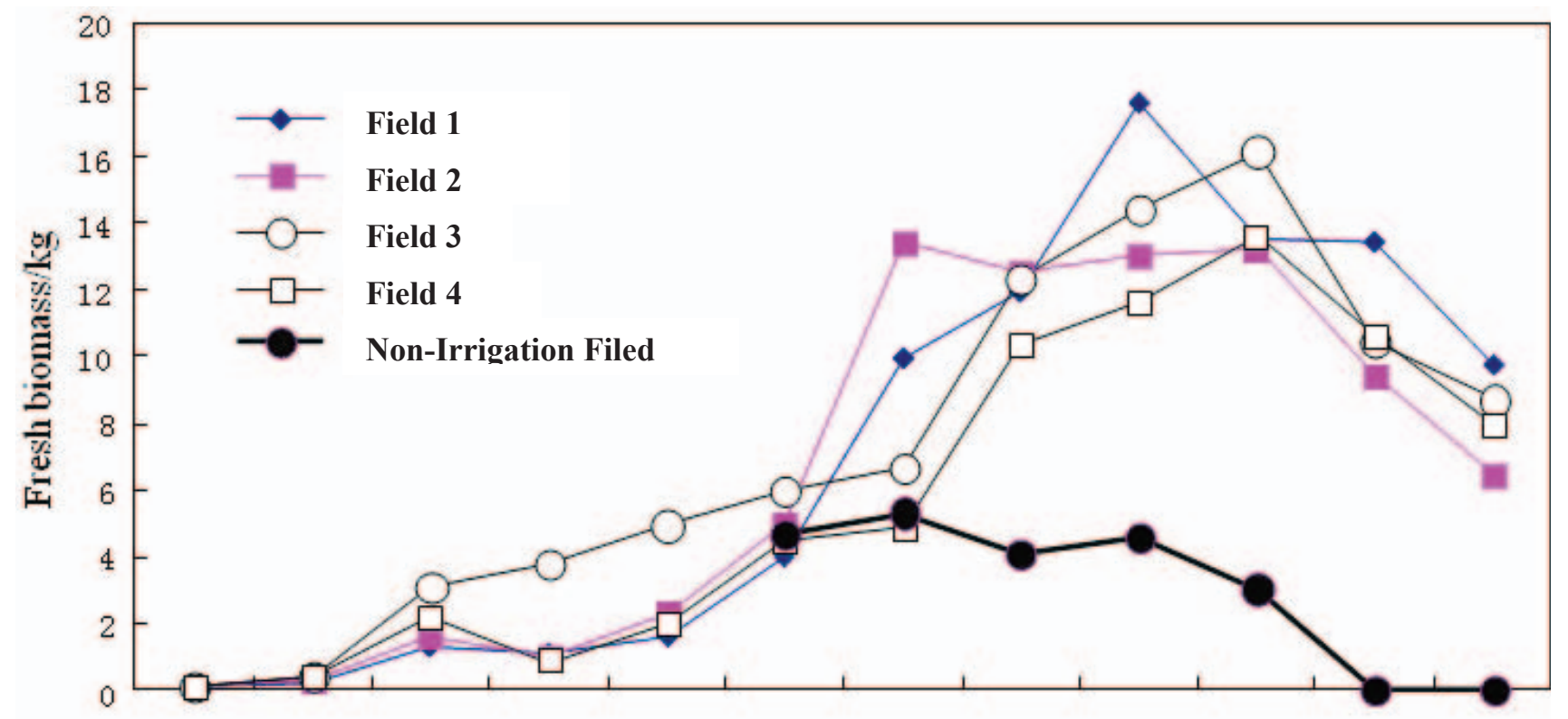

Fig.2 The dynamic of biomass of five wheat fields

\section{METHOD AND CONCLUSION}

The conventional Water-Cloud Model ${ }^{[1]}$ and AIEM surface model ${ }^{[2][3]}$ are combined to form a simple vegetation model, with field samples as inputs, the model simulating results are compared with satellite SAR observed backscatter coefficient at different polarization, results show simulation and observation fit well. Based on this vegetation model, soil moisture and wheat biomass are inversed and compared with in-situ measurements, good results are obtained, which prove that quad-polarized Radarsat-2 image at one acquirement can estimated these surface parameters with good accuracy.

Some important polarimetric parameters and polarimetric response characteristic are also obtained and analyzed, the following conclusions can be drawn by now:

- HH polarization has better sensitivity with soil moisture over wheat covered area, especially when wheat is short.

- Eigen Value $\lambda_{2}$ based on Cloude polarimetric target decomposition ${ }^{[4]}$ is sensitive to soil moisture well, shows its great potential in soil moisture estimation.

- Great scattering mechanism difference of wheat can be seen before and after tassels coming out, Milking Stage has more complex scattering mechanism than Booting Stage, and Scattering Entropy (H) shows good potential in monitoring wheat growing situation.

- VV/VH combination has better relations with biomass due to the structure characteristic at Milking Stage, on the other hand, $\mathrm{HH} / \mathrm{HV}$ combination has better relations with product. 


\section{REFERENCES}

[1] Attema E P, Ulaby F T. Vegetation Modeled As A Water Cloud. Radio Science, Vol. 13 (2): 357 364, 1978

[2] Fung, A. K., Z. Q, Li, and K. S. Chen, Backscattering from a randomly rough dielectric surface, IEEE Transactions on Geoscience and Remote Sensing, vol. GE-30, no. 2, pp.356-369, 1992.

[3] Tzong Dar Wu, K S Chen, Jiancheng Shi. A Transition Model for the Reflection Coefficient in Surface Scattering, IEEE Transactions on Geoscience and Remote Sensing, Vol. 39: 2040-2050, 2001

[4] Cloude S R, Pottier E. A review of target decomposition theorems in radar polarimetry. IEEE Transactions on Geoscience and Remote Sensing, 1996, $34(2): 498-518$ 